\title{
AiMT
}

Advances in Military Technology

Vol. 13, No. 1 (2018), pp. 5-12

ISSN 1802-2308, eISSN 2533-4123

DOI 10.3849/aimt.01170

\section{Deciding DUT Proper Functionality in Non-Metrological Measurements}

\author{
Z. Růžička* \\ Faculty of Military Technology, University of Defence in Brno, Czech Republic
}

The manuscript was received on 14 December 2016 and was accepted after revision for publication on 20 March 2018.

\begin{abstract}
:
Establishing the criterion for whether a device under test (DUT) operates correctly or not is a complex issue. For devices used in a military setting, it is essential to know if the equipment will perform accurately under adverse conditions. In this paper, the example of testing resistive temperature sensors demonstrates how to manage the evaluation of errors expressed as uncertainties and tolerances. These sensors' errors are used to address the total standard uncertainty, to determine the effective degree of freedom and to determine the coverage probability for deriving the coverage factor to establish the Student's quantile as the specific criteria relating to the DUT results.
\end{abstract}

\section{Keywords:}

RTD's class of accuracy, expanded uncertainty, tolerance, standard uncertainty, dynamic error, coverage probability, coverage factor

\section{Introduction}

When evaluating the correct functioning of a sensor or measuring apparatuses, it is a standard procedure to compare the response of a Device Under Test (DUT) with the correct values produced under the specific conditions and appropriate standards. Differences emerge from the resulting DUT response errors. Such measurements are called metrological and can only be conducted in official, authorized laboratories.

The specified conditions generally include a steady-state status and are generally quite conservative, with severely limited variations in parameter values (including measured parameters) and the standard properties. Through calculations, a certain reference point is formulated. If the DUT meets the reference point requirements, it is assumed to function correctly across the specified measuring range even in variations of values affecting the quantities maintained within the limits by the DUT.

\footnotetext{
* Corresponding author: Department of Electrical Engineering, Faculty of Military Technology, University of Defence in Brno, Kounicova 65, 66210 Brno, Czech Republic.

Phone: +420 9734426 45, e-mail: zbynek.ruzicka@unob.cz
} 
Often a researcher or end user needs to take metrology-like measurements for their own purposes, and yet they might face a problem that the manufacturer's conditions for metrological measurement cannot be met. In practice, the problem is in the phrase "under specified conditions". During the test, measurements carried out at measured values within a particular broad range, but not only in the reference point, a more intricate criterion must be determined for making a decision whether the DUT operates properly or not under less controlled conditions. However, meeting such a criterion may be interpreted as a chance without sufficient metrological "certainty". Nevertheless, it can deliver adequate data to distinguish DUTs that most likely do not operate correctly from those working properly with the correct functionality.

The following example outlines a way to formulate the proposed criterion.

\section{Measurements to examine RTDs reliability}

Recently, the Faculty of Military Technology conducted an accelerated lifespan test of resistive temperature detectors (RTDs). Four suits of four models of sensors consisting of 30 pieces each were placed inside a heating/cooling box and were repeatedly stressed by temperature shocks from $-50{ }^{\circ} \mathrm{C}$ to $+180{ }^{\circ} \mathrm{C}$ and vice versa. A reference RTD was located nearby the suits. An autonomous internal system of the box controlled the temperature shifts and at a set target value. A fan was used to circulate air inside the box. The airflow distribution partly depended on shapes of the objects inserted into the box and it was deemed stable for the invariable inner arrangement. Due to the number of sensors, a two-wire connection was chosen for the test.

An appropriate computer programs the assigned target temperatures cyclically. Some of the shock-cycles were "burdening" tests while others involved taking detailed measurements at temperatures at three target values, i.e. $+180{ }^{\circ} \mathrm{C}, 0{ }^{\circ} \mathrm{C}$ and $-50{ }^{\circ} \mathrm{C}$. Upon approaching the target temperature, the response sets of all sensors, including the reference, were scanned and stored. Concurrently, the results were individually compensated for leading wire resistances measured before starting the temperature cycling, i.e. before the proper lifespan test.

Temperature data $\vartheta_{i}$ of $i$-th RTD compared to $\vartheta_{\mathrm{R}}$ of the reference RTD placed in the box nearby the suits of RTDs arrangement results in a temperature indication difference

$$
D_{i}=\vartheta_{i}-\vartheta_{\mathrm{R}}
$$

The difference exists due to measurement errors and it makes a basis for $i$-th RTD's functionality evaluation.

\section{Criterion to identify faulty RTDs}

The key question is, whether an obtained value of the temperature indications' difference is justifiable or not. To establish the criterion, the researcher has to take into account the sources of all participating measurement errors and to process them with respect to their probability distributions.

In many cases, there is an uncertainty or tolerance describing the accepted limits of errors given, but, unfortunately, the information on error probability distributions and / or coverage probabilities relating to the uncertainty or tolerance is often missing. According to the data's behaviour, the researchers can consider the stated figure being either an expanded uncertainty $U_{\mathrm{N}}$ that delimits normally distributed errors (with usual coverage probability of $95 \%$ and coverage factor of 1.96) or a tolerance. The toler- 
ance demarcates errors distributed uniformly within the tolerance zone displayed symmetrically in the indication vicinity so that the errors' probability distribution is a rectangular one with the coverage probability equal to $100 \%$. An appropriate coverage factor equals $\sqrt{ } 3$.

Since the expanded uncertainties and tolerances are subject to different coverage coefficients, standard uncertainties must be used to determine cumulative uncertainty as a combined standard uncertainty (see [1] and [2] for more details). A standard uncertainty $u$ T related to a tolerance $\pm T$ applies.

$$
u_{\mathrm{T}}=\frac{T}{\sqrt{3}} \cong 0.577 \cdot T \text {. }
$$

If the figure above shows the expanded uncertainty $U_{\mathrm{N}}$ with an associated coverage factor of 1.96 , the appropriate standard uncertainty $u_{\mathrm{N}}$ is therefore

$$
u_{\mathrm{N}}=\frac{U_{\mathrm{N}}}{1.96} \cong 0.510 \cdot U_{\mathrm{N}}
$$

\subsection{Error Sources}

Concerning the RTD, two significant sources of thermal error sources may occur: one concerning the RTD itself and the other having a reference to the RTD. As described later, there are also other sources of errors, but these are independent of the sensors, and therefore have little relevance in this context.

A detailed survey of possible RTD partial errors is presented in [3]. Where the total RTD error is customarily specified by limits, i.e. tolerances, quantified as a sum of two components, one of them depending on the presented data (the temperature indication $\vartheta$ ) and the other being fixed for the given RTD type. The established classes of accuracy for platinum resistive sensors, their forms and values are applied in Tab. 1 [4]. No closer specification concerning the sensor's errors are given, except for not exceeding such stated limits. For this reason, these specifications are seen as tolerances, and as such are rectangular to the probability distribution error.

Tab. 1 Classes of accuracy and tolerances of platinum resistive sensors [3]

\begin{tabular}{|c|c|c|}
\hline Class of accuracy & Tolerance at temperature $\vartheta\left[{ }^{\circ} \mathrm{C}\right]$ & Temperature range $\left[{ }^{\circ} \mathrm{C}\right]$ \\
\hline $1 / 10 \mathrm{~B}$ & $\pm(0.03+0.0005 \cdot|\vartheta|)$ & 0 to 100 \\
\hline $1 / 3 \mathrm{~B}$ (or AA) & $\pm(0.10+0.0017 \cdot|\vartheta|)$ & -50 to 250 \\
\hline $\mathrm{A}$ & $\pm(0.15+0.002 \cdot|\vartheta|)$ & -50 to 650 \\
\hline $\mathrm{B}$ & $\pm(0.3+0.005 \cdot|\vartheta|)$ & -200 to 850 \\
\hline $\mathrm{C}$ & $\pm(0.60+0.01 \cdot|\vartheta|)$ & -200 to 850 \\
\hline
\end{tabular}

Commonly, sensors from classes A to $\mathrm{C}$ are used, while the classes of lines 1 and 2 (Tab. 1) are used to apply mostly for metrological purposes. All sensors used in the measurement test described belong to class B and their indications standard uncertainties are, according to Eq. (2),

$$
u_{i} \cong 0.577 \cdot\left(0.3+0.005 \cdot\left|\vartheta_{i}\right|\right)
$$




$$
u_{\mathrm{R}} \cong 0.577 \cdot\left(0.3+0.005 \cdot\left|\vartheta_{\mathrm{R}}\right|\right),
$$

$u_{i}$ denoting the standard uncertainty of the $i$-th RTD's indication and $u_{\mathrm{R}}$ of the RTD reference.

The next source of difference $D_{i}$ total error component stems from thermal inhomogeneity inside the heating / cooling box despite the air being circulated by a fan. Therefore, an RTD and the reference RTD are exposed to (and measure) slightly different temperatures. Owing to the thermal box data, inhomogeneity $\pm H$ is a kind of tolerance and the rectangular distribution is justified here. Therefore, for the corresponding standard uncertainty $u_{\mathrm{H}}$, the following formula can be applied:

$$
u_{\mathrm{H}} \cong 0.577 \cdot H \text {. }
$$

Measuring the RTD's resistance introduces an error due to the connection resistance between an RTD and the measuring instrument. This applies for all RTDs inclusive of the reference one. Although all leading copper-wire resistances at the box temperature $0{ }^{\circ} \mathrm{C}$ were compensated for, their changes occurred due to the wire's temperature variations. In particular, about $1 / 7$ of the wire's length was inside the thermal box and exposed to maximum temperature variations, as it nominally reached $180{ }^{\circ} \mathrm{C}$. In this instance, the rated resulting relative change of the wire's resistance transferred to temperature error is utmost $(1 / 7) \cdot 180 \cdot 0.00383 \approx 0.098{ }^{\circ} \mathrm{C}$ (where 0.00383 is the copper temperature coefficient of resistance) which is about one order less than some of the other errors. Moreover, when establishing the difference $D_{i}$, such errors (of systematic behaviour) of the $i$-th RTD and the reference RTD are partially compensated for. As a result, these errors are negligible.

The same conclusion was reached after consideration of the $D_{i}$ error caused by the additional heating of RTDs by measuring current. Usually, there is not enough information available to calculate this type of error in detail, but it is included in the specifications of a sensor's accuracy through its accuracy class providing that the current value is maintained within the specified interval. Maximum and recommended current is usually predetermined. For this experiment, the recommended current was used. In addition, those small errors of two sensors compensates for in the difference $D_{i}$.

Finally, there is a dynamic error arising due to imperfectly reaching the temperature's steady state when reading the temperature indications of both an RTD and the reference RTD. In reality, one can never achieve an absolute steady state due to small degrees of instability inherent to the box temperature's controlling system. Moreover, inertias (differing each one from another) of the RTDs are active.

As a result, the collected differences $D_{i}$ belonging to the $i$-th RTD are detected during the temperature measuring cycle at variable temperature readings that are similar to, but still different from the target temperature (e.g. $180{ }^{\circ} \mathrm{C}$ ). A difference $D_{i}$ error due to inertia reflects different inertias of sensors, variations of both temperature and airflow inhomogeneity in the box, variations of deviations of reading the temperature from the target one, nonlinearity of the $i$-th RTD and of the reference RTD, as well as some unspecified influences. With respect to these factors, it is advisable to evaluate families of differences $D_{i}$ consecutively collected and coming from different shock-cycles. For establishing the criterion to judge the $n$-th temperature difference, statistical evaluations of a family consisting of the previous $n^{-1}$ elements $D_{i}$ for a given target temperature can provide an estimation, as an average difference $D_{i \text {; }}$ provided that none of the $n-1$ differences was evaluated as being defected. Due to the 
number of random component grounds and according to the Central Limit Theorem, it is justified to expect an approximately normal distribution of the random component values probability and to estimate the variations by means of standard deviation of the

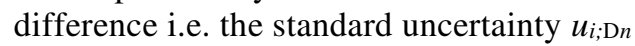

$$
u_{i ; \mathrm{D} n}=\sqrt{\frac{1}{n^{\prime}-2} \sum_{i=1}^{n^{\prime}-1}\left(D_{i ; j}-D_{j ; \mathrm{a}}\right)^{2}},
$$

$n^{\prime}$ denoting the portion of all $n \geq n^{\prime}$ indications that belong to the population of indications satisfying the criterion.

\subsection{The Thermal Difference Total Standard Uncertainty}

The measurement function Eq. (1) has a very simple form and the sensitivity coefficients or partial derivatives $\partial D_{i} / \partial \vartheta_{i}$ and $\partial D_{i} / \partial \vartheta_{\mathrm{R}}$ are equal to 1 . Hence, the cumulated difference or total standard uncertainty is

$$
u_{i ; \mathrm{TD}}=\sqrt{u_{i}^{2}+u_{\mathrm{R}}^{2}+u_{\mathrm{H}}^{2}+u_{i \mathrm{D}}^{2}} .
$$

Using Eq. (1) and Eq. (4) to (7) provides a practical expression to calculate $u_{i ; \mathrm{TD} n}$ applicable to the last, i.e. the $n$-th read temperature indications $\vartheta_{i ; n}$ and $\vartheta_{\mathrm{R} n}$

$u_{i ; \mathrm{TD} n}=\sqrt{0.06+1 \cdot 10^{-3}\left(\left|\vartheta_{i ; n-1}\right|+\left|\vartheta_{\mathrm{R} ; n-1}\right|\right)+8.3 \cdot 10^{-6}\left(\vartheta_{i ; n-1}^{2}+\vartheta_{\mathrm{R} ; n-1}^{2}\right)+\frac{H^{2}}{3}+\frac{1}{n^{\prime}-2} \sum_{i=1}^{n^{\prime}-1}\left(D_{i ; j}-D_{i ; \mathrm{a}}\right)^{2}}$

Using $\vartheta_{i ; n-1}$ and $\vartheta_{\mathrm{R} n-1}$ evaluated positively during the previous stage prevents contaminating $u_{i ; \mathrm{TD} n}$ by values that do not belong to the population. In this regard, the criterion will be predictive by steps.

\subsection{Effective Degree of Freedom}

The effective degree of freedom $v_{i ; \mathrm{TD} n}$ attributed to $u_{i ; \mathrm{TD} n}$ is one of two assessment bases for the value's coverage factor (related to Student's distribution) and further to the expanded uncertainty $U_{i ; \mathrm{TD} n}$, the desired coverage probability is the other base.

In general, $v_{i ; \mathrm{TD} n}$ depends on the standard uncertainties at play and on their degrees of freedom. Their relation is the Welch-Satterthwaite formula [2] according to which is

$$
\mathrm{v}_{i ; \mathrm{TD} n}=\frac{u_{i ; \mathrm{TD} n}^{4}}{\frac{u_{i}^{4}}{\mathrm{v}_{i}}+\frac{u_{\mathrm{R}}^{4}}{\mathrm{v}_{\mathrm{R}}}+\frac{u_{\mathrm{H}}^{4}}{\mathrm{v}_{\mathrm{H}}}+\frac{u_{i ; \mathrm{D} n}^{4}}{v_{i ; \mathrm{D} n}}}
$$
Hence

In this case, the degrees of freedom $v_{i \mathrm{~T}}, v_{\mathrm{R}}$, and $v_{\mathrm{H}}$ equal infinity and $v_{i ; \mathrm{D} n}=n^{\prime}-2$.

$$
v_{i ; \mathrm{TD} n}=\left(n^{\prime}-2\right) \frac{u_{i ; \mathrm{TD} n}^{4}}{u_{i ; s \mathrm{D} n}^{4}}
$$

\subsection{Coverage Probability and Coverage Factor}

Before a derivation of the coverage factor $k$, coverage probability $p$ should be selected and attributed to the demanded reliability of the criterion. Coverage factors that corre- 
spond to the chosen $p$ vary with $v_{i ; \mathrm{TD} n}$ and are equal to Student's fractile $t_{p}(v)$ values that are available in a table (e.g. Tab. G.2 in [2] or Tab. IIa in [5]), being

$$
k_{n}=t_{p}(v)=t_{p}\left(v_{i ; \mathrm{TD} n}\right) .
$$

Handling the data available on-line, it is also possible to calculate the coverage factor $k_{n}$ successively according to $n^{\prime}$ etc. with the appropriate probability $(1+p) / 2$ which corresponds to a symmetrical two-sided confidence interval and to the accepted coverage probability $p$. A spreadsheet calculator can be useful for performing this. (For example, a function T.INV is felicitous for the calculation in Microsoft Excel.) Whilst commonly used probability value of $p=95 \%$ may be rated too low for the purpose, $p=99 \%$ seems to be a more acceptable value and results in a more reliable final DUT decision-making.

\subsection{The Criterion}

Multiplying the $u_{i ; \mathrm{TD} n}$ by $k_{n}$ gives a value of expanded uncertainty

$$
U_{i ; \mathrm{TD} n}=u_{i ; \mathrm{TD} n} \cdot k_{n},
$$

which is believed the magnitude of difference $D_{i ; n}$ should not exceed. Therefore, the $i$-th RTD to meet the test in $n$-th measuring means satisfies the criterion

$$
\left|D_{i ; n}\right| \leq U_{i ; \mathrm{TD} n} \text {. }
$$

\section{Practical Application Suggestions}

From the very beginning of cycling the test, $n$ is very small and the magnitude of $u_{i ; \mathrm{D} n}^{2}$ may strongly dominate the sum of other addends in (9) provided the temperature difference indications fluctuate intensively. Hence, it would lack purpose to use Eq. (13) and (14) for a very small sample size $n$. However, one may evaluate several very initial read differences $D_{i}$ "backwards" by $D_{i ; N}$ after $n$ having reached a high enough value $n=N$ (e.g. 20 to 30 ) provided that all the readings to that point comply with $D_{i ; N}$ and belong to the population. This technique causes no additional problems when managing the collected data off-line, but users should be mindful of this issue when creating a programme for operating on-line. If an indication does not comply with the criterion, it is thought not to belong to the population and needs to be excluded from computing the $U_{i ; \mathrm{TD} n}$.

Conversely, in case the $u_{i ; \mathrm{D} n}{ }^{2}$ is negligible compared to the other addends in Eq. (9), one can find minimal values of $u_{i ; \mathrm{TD} n}$ for the three target temperatures $-50{ }^{\circ} \mathrm{C}$, $0{ }^{\circ} \mathrm{C}$ and $180{ }^{\circ} \mathrm{C}$, respectively: $\min \left\{u_{i ; \mathrm{TD} n\left(-50^{\circ} \mathrm{C}\right)}\right\}=1.02{ }^{\circ} \mathrm{C}, \min \left\{u_{; i \mathrm{TD} n}\left(0^{\circ} \mathrm{C}\right)\right\}=0.900{ }^{\circ} \mathrm{C}$, $\min \left\{u_{i ; \mathrm{TD} n}\left(180^{\circ} \mathrm{C}\right)\right\}=1.67^{\circ} \mathrm{C}$ (with $\left.H=1.5^{\circ} \mathrm{C}\right)$.

Accordingly Eq. (11), the effective degree of freedom rises with $n^{\prime}$ increasing as well, and if $n^{\prime}$ or $u_{i ; \mathrm{TD} n} / u_{i ; \mathrm{D} n}$ is high enough, the $v_{i ; \mathrm{D} n}$ approaches infinity giving $k_{n}=2.576$ for $p=99 \%$. Therefore, minimal values may be evaluated of expanded uncertainties for indications equal to the target temperatures:

$\min \left\{U_{i ; \mathrm{TD} n\left(-50{ }^{\circ} \mathrm{C}\right)}\right\}=2.63{ }^{\circ} \mathrm{C}, \min \left\{U_{i ; \mathrm{TD} n\left(0{ }^{\circ} \mathrm{C}\right)}\right\}=2.32{ }^{\circ} \mathrm{C}, \min \left\{U_{i ; \mathrm{TD} n}\left(180^{\circ} \mathrm{C}\right)\right\}=4.30{ }^{\circ} \mathrm{C}$.

These values are suitable Rules of Thumb for rough estimates. When an RTD complies with these more severe limits, it does so also with the criterion Eq. (14) for every $n^{\prime}$. 


\section{An Illustrative Example}

Fig. 1 shows an example of a resulting measurement record of $\mathrm{RTD}_{18}$ that has not passed the test for the target temperature $0{ }^{\circ} \mathrm{C}$. According to Eq. (9), (11), (12) and (13), the values of the Criterion curve as well as those from the Rule of Thumb curve (with omitting $u_{18 \mathrm{D} n}$ ) for the actual temperature indications were calculated. One-step predictive evaluation was used to exclude indications from the calculation that did not comply with the criterion.
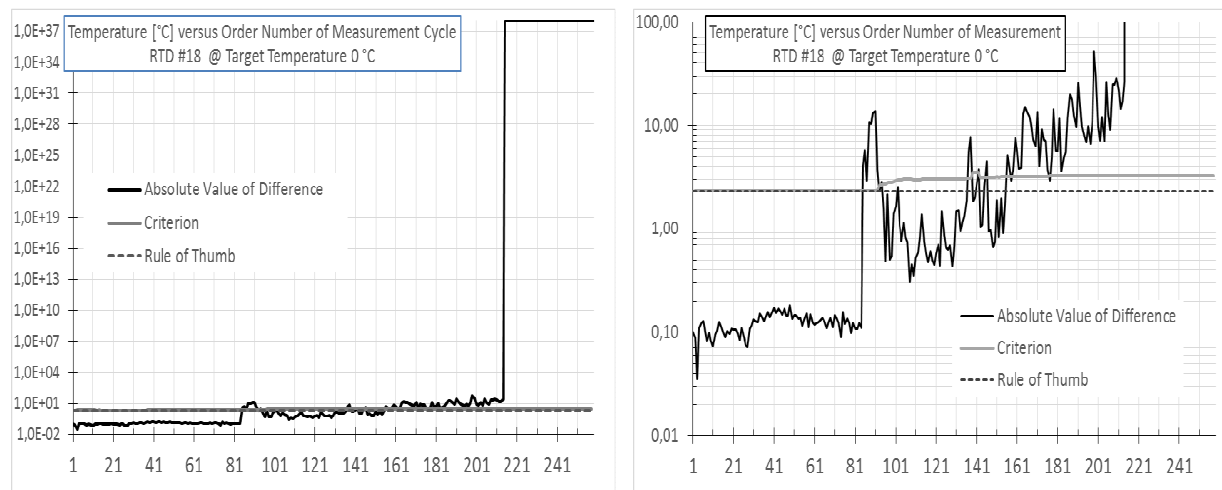

Fig. 1 Graph of absolute value of difference $\vartheta_{18}-\vartheta_{\mathrm{R}}$ (left) and a detail of it (right).

The vertical scales represent the indicated temperature in degrees of Celsius; the horizontal scales denote order numbers of measuring cycles.

Obviously, variations of readings due to the dynamic error discussed in 3.1 were very small here and the value of $u_{i ; \mathrm{D} n}$ was very small until a malfunction of $\mathrm{RTD}_{18}$ manifested. As a result, the Criterion curve almost copies the Rule of Thumb in this part of the graph.

The fault of $\mathrm{RTD}_{18}$ was deemed to be creeping. After several additional loading cycles had passed, its exhibition diminished and the sensor seemed good despite the strong variation in thermal difference. Consequently, the calculated Criterion value rose a little, as the $u_{i ; \mathrm{D} n}$ was no longer negligible. Later, the $\mathrm{RTD}_{18}$ fault rise continued until the sensor circuit disconnected which was a response of the indication limit about $1 \times 10^{37}{ }^{\circ} \mathrm{C}$.

\section{Conclusion}

Rather than continue to be frustrated by DUT errors, this article presents an approach to appreciate the effect of errors accompanying a measurement on the measurement result reliability expressed as an expanded uncertainty attributed to the measurement result.

As outlined in the article, the process was divided into several consecutive steps starting with the measurement function formulation for the quantities present. The next step, which involved revealing all the potential error sources, was critical. After that, every error (given as an expanded uncertainty or tolerance) needed to be converted to standard uncertainty taking into account the error probability distribution. Then, using the appropriate sensitivities of measurement function to their quantities variations, individual error contributions to the standard uncertainty of measurement results could 
be derived. A mutual comparison of these contributions allowed for the exclusion from future computation those whose values were at least two orders in magnitude smaller than others, alternatively. Significant contributions added in the RSS fashion have thus given the resulting standard uncertainty. To designate expanded uncertainty as an indicator of result reliability, its degree of freedom is to be specified using standard uncertainties of single errors and their degrees of freedom. After accepting coverage probability of the measurement result, a coverage factor as the Student's quantile can be found and resulting expanded uncertainty of measurement result can be finally assessed.

\section{Acknowledgement}

The research work presented in this paper has been supported by the Ministry of Defence of the Czech Republic - research project VÝZKUMFVT (DZRO K-217).

\section{References}

[1] TNI 010115 / ISO / IEC Guide 99:2007. Basic and General Concepts and Associated Terms (VIM). ÚNMZ, 2009, 88 p.

[2] ČSN P ENV 13005/ENV 13005:1999. Guide to Expression of Uncertainty in Measurement. ČNI, 2005, 239 p.

[3] Resistance Thermometry: Principles and Applications of Resistance Thermometers and Thermistors. MINCO, 2011, 24 p. Available at <https://www.minco. $\mathrm{com} / \sim / \mathrm{media} /$ files/minco/whitepapers/sensors/resistance-thermometry.ashx $>$.

[4] KREIDL, M. Temperature Measurement - Sensors and Measuring Circuits (in Czech). Prague: BEN, 2005, 239 p. ISBN 80-7300-145-4.

[5] ČSN ISO2854. Statistical Data Interpretation - Estimates and Tests of Mean Values and Scattering (in Czech). ČNI, 1994, 41 p. 